\title{
APPLICATION OF VEHICLE DYNAMIC MODELING IN UAVS FOR PRECISE DETERMINATION OF EXTERIOR ORIENTATION
}

\author{
M. Khaghani ${ }^{a *}$ J. Skaloud ${ }^{a}$ \\ ${ }^{a}$ EPFL ENAC IIE TOPO, Bâtiment GC, Station 18, 1015 Lausanne, Switzerland - (mehran.khaghani, jan.skaloud)@epfl.ch
}

\section{ICWG III/I}

KEY WORDS: Sensor Orientation, Precise Attitude Determination, Navigation, UAV, Corridor Mapping, Vehicle Dynamic Model

\begin{abstract}
:
Advances in unmanned aerial vehicles (UAV) and especially micro aerial vehicle (MAV) technology together with increasing quality and decreasing price of imaging devices have resulted in growing use of MAVs in photogrammetry. The practicality of MAV mapping is seriously enhanced with the ability to determine parameters of exterior orientation (EO) with sufficient accuracy, in both absolute and relative senses (change of attitude between successive images). While differential carrier phase GNSS satisfies cm-level positioning accuracy, precise attitude determination is essential for both direct sensor orientation (DiSO) and integrated sensor orientation (ISO) in corridor mapping or in block configuration imaging over surfaces with low texture. Limited cost, size, and weight of MAVs represent limitations on quality of onboard navigation sensors and puts emphasis on exploiting full capacity of available resources. Typically short flying times (10-30 minutes) also limit the possibility of estimating and/or correcting factors such as sensor misalignment and poor attitude initialization of inertial navigation system (INS). This research aims at increasing the accuracy of attitude determination in both absolute and relative senses with no extra sensors onboard. In comparison to classical INS/GNSS setup, novel approach is presented here to integrated state estimation, in which vehicle dynamic model (VDM) is used as the main process model. Such system benefits from available information from autopilot and physical properties of the platform in enhancing performance of determination of trajectory and parameters of exterior orientation consequently. The navigation system employs a differential carrier phase GNSS receiver and a micro electro-mechanical system (MEMS) grade inertial measurement unit (IMU), together with MAV control input from autopilot. Monte-Carlo simulation has been performed on trajectories for typical corridor mapping and block imaging. Results reveal considerable reduction in attitude errors with respect to conventional INS/GNSS system, in both absolute and relative senses. This eventually translates into higher redundancy and accuracy for photogrammetry applications.
\end{abstract}

\section{INTRODUCTION}

A novel autonomous navigation for unmanned aerial vehicles based on vehicle dynamic model recently introduced by the authors in (Khaghani and Skaloud, 2016), focused on reducing navigation error in GNSS outage conditions. This contribution explores the possibility of using that method in presence of high accuracy GNSS solution to improve attitude determination accuracy for photogrammetry applications, compared to conventional INS/GNSS integration. A shortened introduction on principles of the proposed method is presented in Section 2 and Section 3, while the main part of the contribution is presented in Section 4 and focuses on results and discussions related to attitude determination accuracy in block and corridor mapping scenarios.

\subsection{Motivation}

Employing UAVs for photogrammetry is very popular nowadays (Colomina and Molina, 2014). Using MAVs has considerably decreased cost and effort needed for photogrammetry tasks during the filed operations. On the other hand, observation of camera position/attitude can facilitate the post processing tasks once the photos are taken. In order to achieve ground errors under ground sampling distance (GSD) levels, the level of required accuracy for EO parameters is rather demanding. This is more crucial for DiSO, since any error on EO parameters directly translates into a ground error. For example, an error of $0.05^{\circ}$ in roll or pitch for a flight altitude of $150 \mathrm{~m}$ means a ground error as big as $13 \mathrm{~cm}$.

With high accuracy carrier-phase differential GNSS positioning, the requirements on positioning accuracy are usually met (Rehak

\footnotetext{
${ }^{*}$ Corresponding author
}

et al., 2013). However, achieving the accuracy requirements on attitude is still challenging with MEMS IMUs typically found on MAVs. Any improvement on attitude determination accuracy is directly reflected in the accuracy of final ortho-photo product in case of $\mathrm{DiSO}$, and can reduce the number of required ground control points (GCP) in case of ISO, especially in corridor mapping scenario (Rehak and Skaloud, 2015). For this reason, a novel VDM based navigation method is employed in this research to improve attitude determination for a MAV. Attitude control is absolutely necessary for sensors such as LiDAR and pushbroom scanners, and it is useful/necessary for frame cameras in case of low or no lateral overlap, low number of GCPs, and over low surface texture (i.e., low number of tie points).

Using relative attitude and position data is a rather new approach in airborne mapping (Blázquez, 2008) that has practical application in MAVs (Skaloud et al., 2014). In ISO, it brings some advantages such as elimination of the need for camera-to-IMU boresight calibration and removing the effect of positioning biases if present. For this reason, the simulation results are presented for both absolute and relative attitude determination approaches in current paper.

\subsection{Proposed approach}

The proposed navigation system in (Khaghani and Skaloud, 2016) integrates VDM with inertial navigation for its autonomous part, and GNSS or other aids when available. The main idea behind this concept is to benefit from the available information on vehicle dynamic model and control input within the navigation system that implicitly rejects the physically impossible motions suggested by IMU due to its errors. The proposed solution requires 
no additional sensors compared to conventional INS/GNSS setup. Therefore, its employment represents no additional cost and weight on the platform, which is an important aspect in small UAVs.

The VDM needs to be fed with the control input to the UAV. This information is already available in the control/autopilot system, but it needs to be put in correct time relations with IMU and other measurements in real life implementation. The other input to VDM is the wind velocity. The proposed solution makes it possible to estimate the wind velocity within the navigation filter itself, even in absence of air pressure sensors. This adds certain redundancy to the system in case of air pressure sensor misbehavior or failure when employed.

The VDM requires a proper structure based on the host platform type (fixed wing, multicopter, etc.) and its control actuators, which is well described in the literature (Cook, 2013) (Ducard, 2009). The model parameters depend on the specific platform. They can be either identified and pre-calibrated, or estimated inflight. This capability for online parameter estimation (dynamic model identification) that does not require pre-calibration, minimizes the effort required in design and operation.

The proposed navigation system was shown to significantly improve the accuracy and mitigate the drift of position, velocity, and attitude uncertainty during GNSS signal reception absence ( 2 orders of magnitude for positioning and 1 to 2 orders of magnitude for attitude determination) (Khaghani and Skaloud, 2016). However, the benefits of employing this system are not limited to GNSS outage conditions. Therefore, we investigate attitude determination improvements provided by this approach when high accuracy GNSS solution is available over the entire trajectory as a potential impact on the quality of ISO and especially DiSO with small UAVs.

\section{VEHICLE DYNAMIC MODEL}

A brief description of the model is presented here, along with the key definitions and equations. More details can be found in (Ducard, 2009) and (Khaghani and Skaloud, 2016).

\subsection{Definitions}

Three coordinate frames are considered in this research, "navigation", "body", and "wind" frames. The navigation frame is a local-level frame oriented in north, east, and down directions denoted by $\left(x_{n}, y_{n}, z_{n}\right)$ or $\left(x_{N}, x_{E}, x_{D}\right)$, and considered to be inertial. Definitions of body frame and wind frame $\left(x_{b}, y_{b}, z_{b}\right)$ can be perceived from Figure 1. The rotation matrix to transform vectors from body frame to navigation frame is defined as a function of roll $(\phi), \operatorname{pitch}(\theta)$, and yaw $(\psi)$ angles.

$$
\begin{aligned}
& R_{n}^{b}=R_{1}(\phi) R_{2}(\theta) R_{3}(\psi) \\
& =\left[\begin{array}{ccc}
1 & 0 & 0 \\
0 & \cos \phi & \sin \phi \\
0 & -\sin \phi & \cos \phi
\end{array}\right]\left[\begin{array}{ccc}
\cos \theta & 0 & -\sin \theta \\
0 & 1 & 0 \\
\sin \theta & 0 & \cos \theta
\end{array}\right]\left[\begin{array}{ccc}
\cos \psi & \sin \psi & 0 \\
-\sin \psi & \cos \psi & 0 \\
0 & 0 & 1
\end{array}\right]
\end{aligned}
$$

The wind frame has its first axis in direction of airspeed $\boldsymbol{V}$, and is defined by two angles with respect to body frame, angle of attack $\alpha$ and sideslip angle $\beta$. Velocity of airflow that is due to UAV's inertial velocity $\boldsymbol{v}$ and wind velocity $\boldsymbol{w}$ is denoted by airspeed vector $\boldsymbol{V}$ as

$$
\boldsymbol{v}=\boldsymbol{V}+\boldsymbol{w}
$$

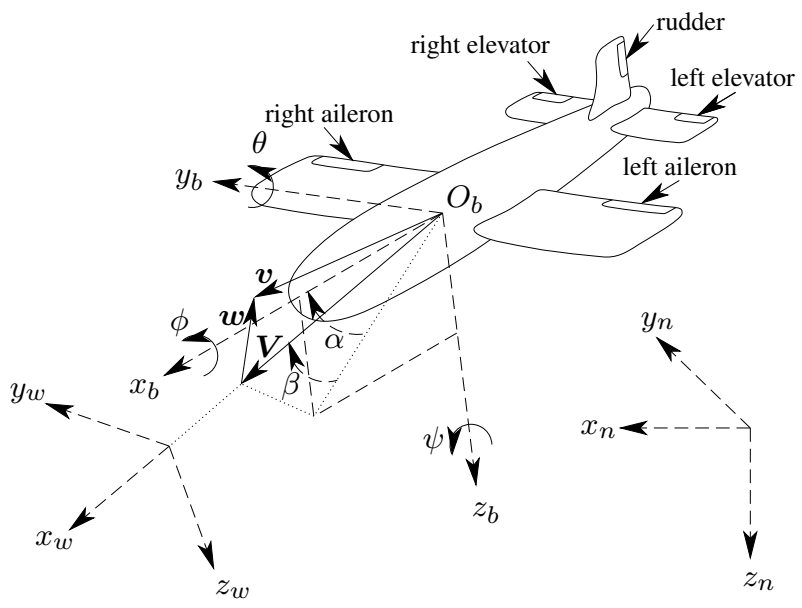

Figure 1: Navigation, body, and wind frames with airspeed $(\boldsymbol{V})$, wind velocity $(\boldsymbol{w})$, and UAV velocity $(\boldsymbol{v})$, as weel as roll $(\phi)$, pitch $(\theta)$, and yaw $(\psi)$

The rotation matrix from body frame to wind frame is defined as a function of angle of attack and sideslip angle as

$$
\begin{aligned}
R_{b}^{w} & =R_{3}(\beta) R_{2}^{T}(\alpha) \\
& =\left[\begin{array}{ccc}
\cos \beta & \sin \beta & 0 \\
-\sin \beta & \cos \beta & 0 \\
0 & 0 & 1
\end{array}\right]\left[\begin{array}{ccc}
\cos \alpha & 0 & \sin \alpha \\
0 & 1 & 0 \\
-\sin \alpha & 0 & \cos \alpha
\end{array}\right] .
\end{aligned}
$$

The density of the air is calculated based on the International Standard Atmosphere model as a function of local pressure and temperature, which can be expressed as functions of the altitude as detailed in (Khaghani and Skaloud, 2016).

\subsection{Dynamic model}

The VDM employed in this research is based on rigid body dynamics for a fixed wing UAV, considering polynomial models for aerodynamic forces and moments (Ducard, 2009). The state vector $\boldsymbol{X}_{n}=\left[x_{N}, x_{E}, x_{D}, v_{x}^{b}, v_{y}^{b}, v_{z}^{b}, \phi, \theta, \psi, \omega_{x}, \omega_{y}, \omega_{z}, n\right]^{T}$, control input vector $\boldsymbol{U}=\left[n_{c}, \delta_{a}, \delta_{e}, \delta_{r}\right]^{T}$, and wind velocity vector $\boldsymbol{w}=\left[w_{N}, w_{E}, w_{D}\right]^{T}$ are related via the dynamic model of the form $\dot{\boldsymbol{X}}_{n}=f\left(\boldsymbol{X}_{n}, \boldsymbol{U}, \boldsymbol{w}\right)$. Components of UAV velocity vector $\boldsymbol{v}$ in body frame are represented by $v_{x}^{b}, v_{y}^{b}$, and $v_{z}^{b}$, while $\omega_{x}^{b}, \omega_{y}^{b}$, and $\omega_{z}^{b}$ denote the rate of change for roll, pitch, and yaw, respectively. Deflections of aileron, elevator, and rudder are denoted by $\delta_{a}, \delta_{e}$, and $\delta_{r}$, respectively. Propeller speed is denoted by $n$, where $n_{c}$ shows the commanded value for that, and $\tau_{n}$ is the time constant for its dynamics. Kinematic equations, Newton's equations of motion, and a first order model for propeller dynamics form the vehicle dynamic model as

$$
\left[\begin{array}{c}
\dot{x}_{N} \\
\dot{x}_{E} \\
\dot{x}_{D}
\end{array}\right]=R_{b}^{n}\left[\begin{array}{c}
v_{x}^{b} \\
v_{y}^{b} \\
v_{z}^{b}
\end{array}\right]
$$

$$
\begin{aligned}
{\left[\begin{array}{c}
\dot{v}_{x}^{b} \\
\dot{v}_{y}^{b} \\
\dot{v}_{z}^{b}
\end{array}\right]=\left[\begin{array}{c}
-g \sin \theta \\
g \sin \phi \cos \theta \\
g \cos \phi \cos \theta
\end{array}\right] } & +\frac{1}{m}\left[\left(\begin{array}{c}
F_{T} \\
0 \\
0
\end{array}\right)+R_{w}^{b}\left(\begin{array}{c}
F_{x}^{w} \\
F_{y}^{w} \\
F_{z}^{w}
\end{array}\right)\right] \\
& -\left[\begin{array}{c}
\omega_{y} v_{z}^{b}-\omega_{z} v_{y}^{b} \\
\omega_{z} v_{x}^{b}-\omega_{x} v_{z}^{b} \\
\omega_{x} v_{y}^{b}-\omega_{y} v_{x}^{b}
\end{array}\right]
\end{aligned}
$$




$$
\begin{gathered}
{\left[\begin{array}{c}
\dot{\phi} \\
\dot{\theta} \\
\dot{\psi}
\end{array}\right]=R_{\omega}\left[\begin{array}{l}
\omega_{x} \\
\omega_{y} \\
\omega_{z}
\end{array}\right], R_{\omega}=\left[\begin{array}{ccc}
1 & \tan \theta \sin \phi & \tan \theta \cos \phi \\
0 & \cos \phi & -\sin \phi \\
0 & \sin \phi / \cos \theta & \cos \phi / \cos \theta
\end{array}\right]} \\
{\left[\begin{array}{c}
\dot{\omega}_{x} \\
\dot{\omega}_{y} \\
\dot{\omega}_{z}
\end{array}\right]=\left(I^{b}\right)^{-1}\left(\left[\begin{array}{c}
M_{x}^{b} \\
M_{y}^{b} \\
M_{z}^{b}
\end{array}\right]-\left[\begin{array}{c}
\omega_{x} \\
\omega_{y} \\
\omega_{z}
\end{array}\right] \times I^{b}\left[\begin{array}{l}
\omega_{x} \\
\omega_{y} \\
\omega_{z}
\end{array}\right]\right)} \\
\dot{n}=-\frac{1}{\tau_{n}} n+\frac{1}{\tau_{n}} n_{c},
\end{gathered}
$$

where $m$ and $I^{b}$ denote mass and moments of inertia matrix of the UAV, respectively. The four aerodynamic forces and the three aerodynamic moments are expressed as polynomial functions of navigation states, control inputs, wind velocity, and physical properties of the UAV called dynamic model parameters hereafter. The aerodynamic forces include:

- "thrust force" as $F_{T}=f\left(\rho, V, D, n, C_{F_{T} \ldots}\right)$

- "drag force" as $F_{x}^{w}=f\left(\rho, V, S, C_{F_{x} \ldots}, \alpha, \beta\right)$

- "lateral force" as $F_{y}^{w}=f\left(\rho, V, S, C_{F_{y} \ldots,}, \beta\right)$

- "lift force" as $F_{z}^{w}=f\left(\rho, V, S, C_{F_{z} \ldots}, \alpha\right)$

The aerodynamic moments include:

- "roll moment" as $M_{x}^{b}=f\left(\rho, V, S, b, C_{M_{x} \ldots}, \delta_{a}, \beta, \omega_{x}, \omega_{z}\right)$

- "pitch moment" as $M_{y}^{b}=f\left(\rho, V, S, \bar{c}, C_{M_{y} \ldots}, \delta_{e}, \alpha, \omega_{y}\right.$

- "yaw moment" as $M_{z}^{b}=f\left(\rho, V, S, b, C_{M_{z} \ldots}, \delta_{r}, \omega_{z}, \beta\right)$

Propeller diameter is denoted by $D$, and $S, b$, and $\bar{c}$ represent wing surface, wing span, and mean aerodynamic chord, respectively. Density of air is shown by $\rho$, and $C$...'s represent aerodynamic coefficients for associated force and moment components. The vehicle dynamic model parameters are collected in (9).

\section{FILTERING METHODOLOGY}

An extended Kalman filter (Gelb, 1974) is chosen to serve as the navigation filter in this research, which is detailed in this section.

\subsection{Scheme}

The proposed navigation system utilizes VDM as main process model within a differential navigation filter. As depicted in Figure 2, VDM provides the navigation solution, which is updated by the navigation filter based on available measurements. Hence, IMU output is treated as a measurement within the navigation filter, just the way GNSS observations are, whenever they are available. Any other available sensory data, such as altimeter or magnetometer output, can also be integrated within the navigation filter as additional observations. VDM is fed with the control input of the UAV, which is commanded by the autopilot and therefore available. Other needed input is the wind velocity as an input, which can be estimated either by the aid of airspeed sensors, or within the navigation system with no additional sensors needed. The latter approach is investigated here. Finally, the parameters of VDM are required within the navigation filter. Pre-calibration of these parameters as fixed values is an option. However, to increase the flexibility, as well as the accuracy of the proposed approach while minimizing the design effort, an online parameter estimation/refinement is implemented. Last but not least, IMU errors are also modeled and estimated within the navigation system as additional filter states.

\subsection{State space augmentation}

The augmented state vector includes the navigation states $\boldsymbol{X}_{n}$, the UAV dynamic model parameters $\boldsymbol{X}_{p}$ excluding mass $(m)$ and moments of inertia $\left(I_{x}, I_{y}, I_{z}, I_{x z}\right)$, the IMU error terms $\boldsymbol{X}_{e}$, and the wind velocity components $\boldsymbol{X}_{w}$.

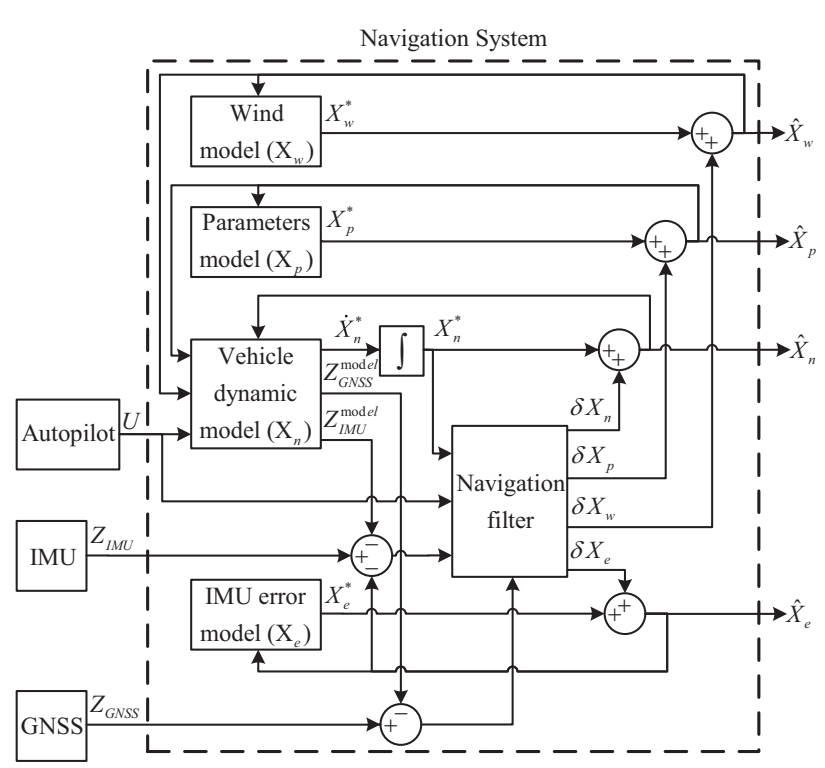

Figure 2: Navigation system architecture

The dynamic model parameters are included in a $26 \times 1$ state vector as in (9), and modeled as constant parameters with initial uncertainties. Description of these parameters is provided in the nomenclature, and the numerical values used in simulation can be found in (Ducard, 2009).

$$
\boldsymbol{X}_{p}=\left[\begin{array}{ccccccc}
S, & \bar{c}, & b, & D, & C_{F_{T} 1}, & \ldots \\
\ldots & C_{F_{T} 2}, & C_{F_{T} 3}, & C_{F_{z} 1}, & C_{F_{z} \alpha}, & C_{F_{x} 1}, & \ldots \\
\ldots & C_{F_{x} \alpha}, & C_{F_{x} \alpha 2}, & C_{F_{x} \beta 2}, & C_{F_{y} 1}, & C_{M_{x} a}, & \ldots \\
\ldots & C_{M_{x} \beta}, & C_{M_{x} \tilde{\omega}_{x}}, & C_{M_{x} \tilde{\omega}_{z}}, & C_{M_{y} 1}, & C_{M_{y} e}, & \ldots \\
\ldots & C_{M_{y} \alpha}, & C_{M_{y} \tilde{\omega}_{y}}, & C_{M_{z} \delta_{r}}, & C_{M_{z} \beta}, & C_{M_{z} \tilde{\omega}_{z}}, & \ldots \\
\ldots & \tau_{n} & & & &
\end{array}\right]^{T}
$$

Mass and moments of inertia are not included in this vector, since they appear as scaling factors in equations of motion and therefore they are completely correlated with the already included coefficients of aerodynamic forces and moments.

The error in each accelerometer and gyroscope inside the IMU is modeled as a random walk $\left(b_{r w}^{\ldots}\right)$ process. Therefore, the IMU error terms vector is defined as

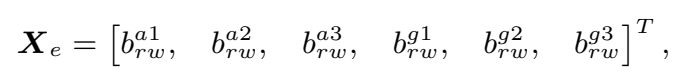

where $a i$ and $g i$ superscripts denote the $i$-th accelerometer and gyroscope, respectively. This model has been found sufficient for the low-cost IMU in consideration, but can be extended as needed.

The wind velocity is stated as a vector in local (navigation) frame consisting of the three components of wind velocity in north, east, and down directions.

$$
\boldsymbol{X}_{w}=\left[w_{N}, w_{E}, w_{D}\right]^{T}
$$

Wind velocity is also modeled as a random walk process.

\subsection{Errors and uncertainties}

For the purpose of simulation, a MEMS-grade IMU is considered. Random biases with standard deviations of $8 \mathrm{mg}$ for accelerometers and $720^{\circ} / \mathrm{hr}$ for gyroscopes are considered, accompanied by white noise and first order Gauss-Markov processes. GNSS error is modelled as independent white noise sig- 
nals for each channel (north, east, down), with standard deviations of $3 \mathrm{~cm}$ for horizontal components and $5 \mathrm{~cm}$ for vertical one. The sampling frequency is $100 \mathrm{~Hz}$ for IMU and $5 \mathrm{~Hz}$ for GNSS measurements.

In terms of initialization errors, random errors are considered for different runs of the Monte-Carlo simulations with standard deviations of $1 \mathrm{~m}$ for each position component, $1 \mathrm{~m} / \mathrm{s}$ for each velocity component, $3^{\circ}$ for roll and pitch, $5^{\circ}$ for yaw, $1^{\circ} / \mathrm{s}$ for rotation rates, and $15 \mathrm{rad} / \mathrm{s}$ for propeller speed. The errors considered for the UAV dynamic model parameters $\left(\boldsymbol{X}_{p}\right)$ are randomly distributed with a standard deviation of $10 \%$.

More details on the process model noise, observation noise, and initial uncertainties can be found in (Khaghani and Skaloud, 2016).

\section{MONTE CARLO SIMULATIONS}

Proof of the proposed concept is performed via Monte Carlo simulation study in several different situations. To make the simulations realistic, errors are introduced to all the a-priori information available to the navigation system, such as initial values of states, dynamic model parameters, and error statistics of IMU and GNSS measurements. Also, real 3D wind velocity data (KNMI and Alterra, 2012) is used in simulations. Results of Monte Carlo simulations on two sample trajectories are presented and analyzed in this paper, one for block imaging and the other for corridor mapping. The reference trajectories for block imaging and corridor mapping are depicted in Figure 3 and Figure 4, respectively.

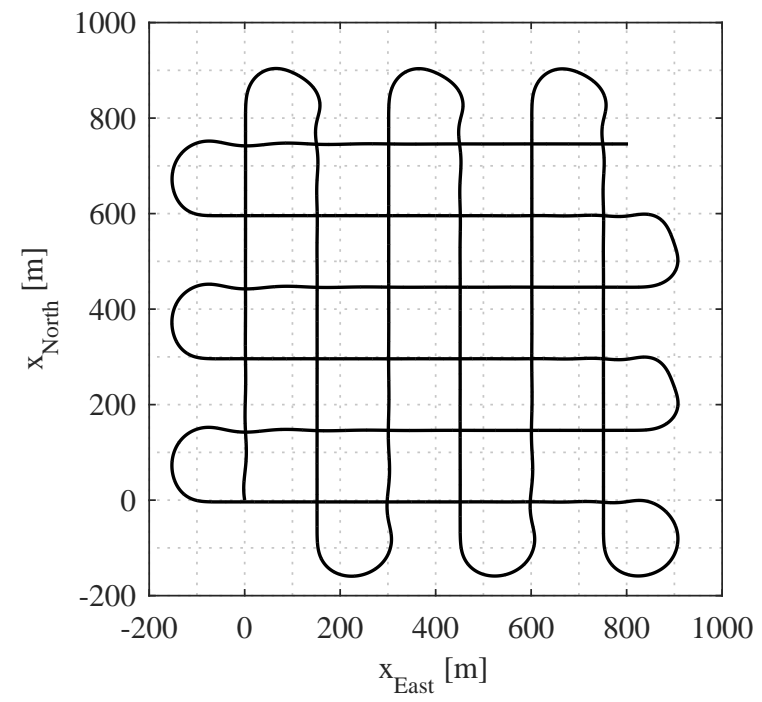

Figure 3: Block imaging trajectory

The Monte Carlo simulation on each trajectory consists of 100 individual runs with different realizations of random errors in sensor data, initialization, and VDM parameters. Table 1 summarizes the results of attitude determination for both trajectories. The attitude errors are calculated based on taking the root mean square (RMS) of errors for all the 100 runs, which is then averaged over the whole trajectory. The numbers on top in each cell show the values for absolute attitude errors, while the numbers in parenthesis show the values for relative ones. The time step between consecutive images for relative attitude calculations is considered to be $2 \mathrm{~s}$. In order to get more realistic results and get closer to real application scenarios in case of block imaging, the data on "turning parts" of trajectory are excluded from averaging

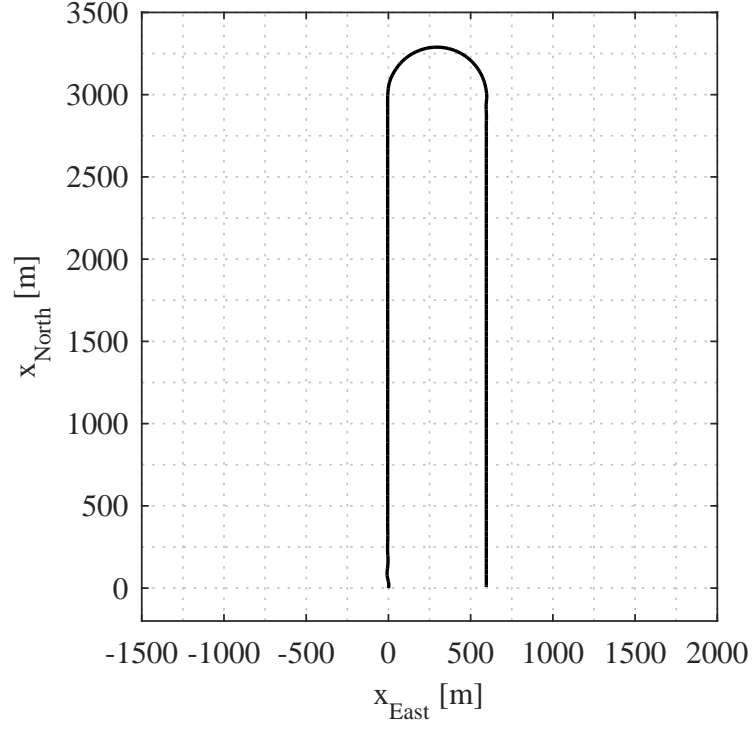

Figure 4: Corridor mapping trajectory

process as the optical instrument would not be used during such maneuvers. Also, a short part at the beginning of each of the two trajectories is omitted as it is predominately influenced by initialization errors and yet unstable gain within the filter. There are a number of interesting facts that can be concluded from the results in Table 1, some of which are discussed in the rest of this section.

\begin{tabular}{c|c|c|c|}
\cline { 2 - 4 } \multicolumn{1}{c}{} & \multicolumn{3}{c|}{$\begin{array}{c}\text { Mean error [deg] } \\
\text { absolute (relative) }\end{array}$} \\
\cline { 2 - 4 } \multicolumn{1}{c|}{} & Roll & Pitch & Yaw \\
\hline Block imaging: & 0.026 & 0.026 & 0.047 \\
\hline \multirow{2}{*}{ INS/GNSS } & $(0.039)$ & $(0.039)$ & $(0.017)$ \\
\hline VDM/INS/GNSS & 0.003 & 0.019 & 0.026 \\
& $(0.006)$ & $(0.006)$ & $(0.013)$ \\
\hline Corridor mapping: & \multicolumn{3}{|c|}{} \\
\hline \multirow{2}{*}{ INS/GNSS } & 0.026 & 0.026 & 0.146 \\
& $(0.039)$ & $(0.039)$ & $(0.037)$ \\
\hline \multirow{2}{*}{ VDM/INS/GNSS } & 0.003 & 0.021 & 0.034 \\
& $(0.004)$ & $(0.007)$ & $(0.013)$ \\
\hline
\end{tabular}

Table 1: Mean error in attitude determination

First thing to notice here is the improvement in attitude estimation provided by VDM/INS/GNSS integration compared to conventional INS/GNSS. Table 2 reveals error reductions in percentages. The combined effect of roll and pitch errors, which is calculated as the norm of the two $\left(\sqrt{(\text { roll error })^{2}+{\text { (pitch error })^{2}}^{2}}\right.$, has been reduced by $47 \%$ and $41 \%$ for absolute case in block imaging and corridor mapping, respectively. At the flight altitude of $150 \mathrm{~m}$, the ground error in DiSO due to this combined effect is equivalently reduced from $9.6 \mathrm{~cm}$ to $5.1 \mathrm{~cm}$ for block imaging, and from $9.6 \mathrm{~cm}$ to $5.6 \mathrm{~cm}$ for corridor mapping. The error reduction for yaw has been $45 \%$ and $77 \%$ for block imaging and corridor mapping, respectively.

For relative attitude determination, the error reduction in combined roll and pitch effect is almost two times greater, being $85 \%$ for both trajectories. For yaw on the other hand, the error reduction is less in both cases, namely $22 \%$ for block imaging and $66 \%$ for corridor mapping.

As can be expected, accurate estimation of yaw is more challenging in corridor mapping compared to block imaging. The reason 


\begin{tabular}{|l|c|c|}
\cline { 2 - 3 } \multicolumn{1}{c|}{} & $\begin{array}{c}\text { Block imaging } \\
\text { absolute (relative) }\end{array}$ & $\begin{array}{c}\text { Corridor mapping } \\
\text { absolute (relative) }\end{array}$ \\
\hline Roll & $87(85)$ & $88(91)$ \\
\hline Pitch & $27(85)$ & $19(81)$ \\
\hline Roll+Pitch & $47(85)$ & $41(85)$ \\
\hline Yaw & $45(22)$ & $77(66)$ \\
\hline
\end{tabular}

Table 2: Percentage of improvement in attitude determination by VDM/INS/GNSS integration compared to INS/GNSS

is lack of enough maneuvers and trajectory dynamic, which diminishes the opportunity of correcting yaw based on GNSS observations within the navigation filter. For conventional INS/GNSS integration, yaw error for corridor mapping is 3.1 times bigger than block imaging, while this ratio is only 1.3 for VDM/INS/GNSS, which reveals much less sensitivity to maneuvers in yaw estimation accuracy. This can be especially important for DiSO, since any error on yaw directly degrades the accuracy of final ortho-photos. For roll and pitch, both INS/GNSS and VDM/INS/GNSS methods show little or no sensitivity to maneuvers.

In ISO, provided that there is sufficient forward overlap between consecutive images and there is enough texture visible in images, image measurements can control pitch and yaw errors to a good extent. Therefore, in applications where there is low side overlap, such as corridor mapping, the most important contribution of aerial attitude control is in roll angle (Rehak and Skaloud, 2015). The proposed VDM/INS/GNSS integration has reduced the roll error 8.2 times for absolute and 10.8 times for relative approach in corridor mapping.

Finally, for both integration methods and for both trajectories, the real value of relative attitude estimation becomes evident in estimation of yaw with 2 to 3.9 times improvements. However, one should notice that in real implementation, the relative approach is capable of removing camera-to-IMU boresight, therefore further improvement in all the three attitude parameters may be expected compared to simulated results in this paper.

\section{CONCLUSION AND PERSPECTIVES}

A novel method to perform autonomous navigation and sensor integration for UAVs was recently introduced by the authors. This method is based on integrating vehicle dynamic model within navigation system, and has been already proven to be very effective in reducing navigation solution drift during GNSS outages. In current paper, the application of this method for photogrammetry applications has been investigated, estimating attitude parameters, while considering the availability of high accuracy GNSS positioning in two trajectories, one for block imaging and another for corridor mapping.

Results of 100 realizations in a Monte Carlo simulation showed considerable error reductions for both absolute and relative attitude determination and for both trajectories. For example, at flight altitude of $150 \mathrm{~m}$ and in case of absolute attitude determination, the improved attitude determination through VDM/INS/GNSS integration would reduce the ground error in DiSO due to combined effect of roll and pitch errors from $9.6 \mathrm{~cm}$ to $5.1 \mathrm{~cm}$ for block imaging, and from $9.6 \mathrm{~cm}$ to $5.6 \mathrm{~cm}$ for corridor mapping. This reduction was almost two times more for roll and pitch in case of relative attitude estimation, but less for yaw.

In conventional INS/GNSS integration, yaw determination is highly sensitive to maneuvers and trajectory dynamic in horizontal plane, as it needs enough dynamic to become observable within the navigation filter. For the two trajectories in this paper, the error in yaw estimation by INS/GNSS was 3.1 times more for corridor mapping compared to block imaging. Such sensitivity is noticeably less for VDM/INS/GNSS, with the same ratio being only 1.3 , which can be considered as an important advantage of this method, especially for DiSO.

Based on simulation results in this paper, the benefit of relative attitude estimation compared to absolute attitude estimation is more evident for yaw. This holds for both conventional and proposed integration methods, and for both trajectories. However, further improvements are expected from relative approach for all $\mathrm{EO}$ parameters in real implementation, since it is capable of removing camera-to-IMU boresight, uncertainty of which is not included in simulations within this paper.

Further development of current work will include studies on proposed navigation system in real scenarios. Technical and perhaps scientific challenges can be expected in real implementation. Proper time stamping of all sensor observations and scaling the control input signals are examples of technical challenges. On the scientific part, the main challenges will probably be related to unmodeled dynamics and disturbances, and the inclusion of additional effects, such as sensor misalignments, actuator dynamics, UAV body elasticity, and asymmetric mass distribution.

\section{REFERENCES}

Blázquez, M., 2008. A new approach to spatio-temporal calibration of multi-sensor systems. The International Archives of the Photogrammetry, Remote Sensing and Spatial Information Sciences 37, pp. 481-486.

Colomina, I. and Molina, P., 2014. Unmanned aerial systems for photogrammetry and remote sensing: A review. ISPRS Journal of Photogrammetry \& Remote Sensing 92, pp. 79-97.

Cook, M. V., 2013. Flight dynamics principles a linear systems approach to aircraft stability and control. ButterworthHeinemann, Waltham, MA.

Ducard, G. J., 2009. Fault-tolerant fight control and guidance systems: Practical methods for small unmanned aerial vehicles. Springer, London.

Gelb, A. (ed.), 1974. Applied optimal estimation. M.I.T. Press, Cambridge, Massachusetts, London.

Khaghani, M. and Skaloud, J., 2016. Autonomous Vehicle Dynamic Model Based Navigation for Small UAVs. NAVIGATION, Journal of the Institute of Navigation (In Print).

KNMI and Alterra, 2012. Data provided by KNMI and Alterra, under the Consortium agreement on Cabauw Experimental Site for Atmospheric Research (CESAR), www.cesar-database.nl.

Rehak, M. and Skaloud, J., 2015. Fixed-wing micro aerial vehicle for accurate corridor mapping. ISPRS Annals of the Photogrammetry, Remote Sensing and Spatial Information Sciences II-1/W1, pp. 23-31.

Rehak, M., Mabillard, R. and Skaloud, J., 2013. A micro-UAV with the capability of direct georeferencing. The International Archives of the Photogrammetry, Remote Sensing and Spatial Information Sciences pp. 317-323.

Skaloud, J., Rehak, M. and Lichti, D., 2014. Mapping with MAV: Experimental study on the contribution of absolute and relative position control. The International Archives of the Photogrammetry, Remote Sensing and Spatial Information Sciences 40-3/W1, pp. 123-129. 